\title{
Modelaje Geoquímico de la Manifestación Volcanico-Exhalativa Submarina "Los Tramojos" en el Complejo Vulcanógeno Sedimentario de Cuba Central.
}

Ricardo A. Valls, Valls Geoconsultant, 1008-299 Glenlake Ave, Toronto, ON, M6P 4A6, Canada. E-mail: vallsgvg@gmail.com

\section{DOI:}

\begin{abstract}
We studied an outcrop of massive iron-copper-zinc sulphides in Cretaceous volcanogenic intermediate to felsic rocks, located to the south of the village Los Tramojos, in the province of Sancti Spiritus, in Central Cuba. It is an artificial cut of dacitic tuffs and volcanic breccias with a strong mineralized area, dark in color, containing pyrite, magnetite, barite, sphalerite, bornite and others copper sulphides, as well as apatite, galenita and chalcopyrite. This area includes another tectonic zone with limonitic, hematitic, zeolitic and chloritic alterations, in contact with tuffs and non-mineralized and fresh volcanic breccias. It is a manifestation of a submarine volcanicexhalative type. The mineralized area described above are very like the so call "Black Ores" of the Kuroko type of deposits. We investigated an area of 3,000 m2 using a soil grid of $25 \times 10$ meters, with some lithogeochemical samples. This study allowed to determine the correlation coefficients characteristic of the ore zone. We recommend the use of these correlation coefficients, combined with geophysical and perforation work, to check ore potential of the Sector "Los Tramojos".
\end{abstract}

Key words: soil sampling, Kuroko, black ores, Central Cuba, correlation coefficient, submarine volcanic-exhalative.

\section{Resumen}

Se estudia una manifestación masiva de sulfuros de hierro-cobre-cinc en rocas cretácicas, volcanógenas, de caracter intermedio a básico. Dicha manifestación se ubica al Sur del poblado Los Tramojos, Provincia de Sancti Spíritus, en Cuba Central. La misma aflora en un corte artificial compuesto por tobas dacíticas y brechas volcánicas que permite observar la existencia de una zona fuertemente mineralizada, de color oscuro, conteniendo principalmente pirita, magnetita, barita, esfalerita, bornita y otros sulfuros de cobre, así como apatito, galenita y calcopirita. Esta zona se acompaña de otra altamente tectonizada, limonitizada, hematizada y localmente zeolitizada y 
cloritizada, en contacto con tobas y brechas volcánicas no mineralizadas y poco alteradas. Se trata de una manifestación del tipo volcánico-exhalativa submarina, coincidiendo la zona mineralizada antes descrita con las llamadas "menas negras" de los yacimientos tipo Kuroko. Para el estudio del sector, se definió un área de $3,000 \mathrm{~m}^{2}$, la cual fué investigada por una red metalométrica de 25 x 10 metros, combinada con algunas muestras litogeoquímicas. Dicho estudio permitió determinar -entre otros factores de interés- los coeficientes correlacionales característicos de la zona menífera y de la zona estéril. El uso de dichos coeficientes correlacionales, aplicados a un muestreo metalométrico sub-regional a escala mediana, acompañado de trabajos geofísicos y de perforación, permitirán una mejor evaluación de la potencialidad menífera del Sector "Los Tramojos".

Palabras claves: muestreo de suelos, Kuroko, menas negras, Cuba Central, coeficiente de correlación, Volcanógeno-exhalativa submarina.

\section{Introducción}

Las formaciones vulcanógeno-sedimentarias del Cretácico ocupan un porciento considerable del área total de afloramientos en Cuba Central. La potencialidad metalogénica de dichas formaciones ha sido enfatizada en varios informes geológicos regionales (A.C.C. y A.C.P. c 1970; M.I.B. 1988), por sólo mencionar dos de los más completos.

El sector de estudio fue ubicado por el técnico Ramón Valdivia y por el autor durante 1988, al cumplimentar muestreos geoquímicos regionales correspondientes al Proyecto TécnicoEconómico "Levantamiento Geológico 1:50 000 Las Villas III" al Sur del poblado Los Tramojos, en la Provincia de Sancti Spíritus, Cuba Central. Al estudiar un corte artificial en un pequeño domo volcánico paralelo al arroyo "Los Tramojos", pudimos constatar la presencia de barita, pirita, sulfuros de cobre y tal abundancia de magnetita que alteraba en varios grados el norte de nuestras brújulas al acercarnos al corte.

El relieve de la zona es ondulado producto de la existencia en el área de varias elevaciones (posiblemente otros domos volcánicos), así como a la acción erosiva del arroyo Los Tramojos, el cual se precipita desde más de 10 metros de altura en un valle profundo y estrecho en forma de "U" y, finalmente, a la actividad del hombre quien ha realizado numerosas excavaciones con vistas a embalsar el agua del arroyo con fines agrícolas, principal renglón económico del área.

Las cotas máximas varían entre los 25 y los 30 metros y el clima es el característico de las llanuras tropicales.

Luego de detectada la manifestación, realizamos un muestreo de suelos en un área de 3,000 $\mathrm{m}^{2}$ sobre el domo volcánico, utilizando una red de 25 x 10 metros sin apoyo topográfico, así como otros muestreos litogeoquímicos directamente en el corte.

El presente trabajo resume los resultados de las investigaciones realizadas y da orientaciones acerca de la continuación de las investigaciones para delimitar el cuerpo mineral detectado, con la ayuda de diferentes coeficientes correlacionales (C.C.).

En la elaboración de este fueron utilizados varios softwares, entre los que podemos destacar SYSTAT y Excel en el procesamiento estadístico; SURFER y CORRELDRAW en la confección de los gráficos y MS Word en la edición del texto. 
Teniendo en cuenta los factores geológicos, tectónicos y mineralógicos, es posible identificar la zona mineralizada con las llamadas "menas negras" de los yacimientos del tipo Kuroko, constituyendo por lo tanto esta investigación el primer estudio geoquímico que de estas manifestaciones se hace en Cuba.

\section{Geología regional}

El área de los trabajos se ubica en la Cuenca Central Cubana. La misma limita al Norte y al Sur con las Cuencas Norte Cubana y Ana María, respectivamente; al Este con el horst-anticlinorio Camagüey, y al Oeste y Noroeste con el macizo metamórfico del Escambray y las zonas estructurofaciales Zaza y Placetas. Se trata de un graben escalonado con espesores de hasta 5 kilómetros mapeado por métodos geofísicos de superficie, principalmente gravimetría, y cortado en toda su potencia por 6 pozos profundos [Algodones $1(1,155 \mathrm{~m})$, Adelaida $1(1,390 \mathrm{~m})$, Vega Grande $(1,585 \mathrm{~m})$, Gabrieles $2(1,680 \mathrm{~m})$, Morón Norte $1(1,820 \mathrm{~m})$ y Jatibonico $78(3,200 \mathrm{~m})]$, de acuerdo con datos correspondientes al año 1990.

Según Milián García $(1988,18)$, las formaciones litoestratigráficas de la Cuenca Central se pueden agrupar en cuatro formaciones meso-cenozoicas.

La base de la Cuenca la componen serpentinitas, basaltos alterados, espilitas, cataclasitas diabásicas y mezclas tectónicas de otras formaciones magmáticas, complicadas por la presencia de secuencias del arco volcánico. Se consideran estas rocas como restos de una corteza oceánica antigua con una edad de emplazamiento tectónico referida al Cretácico Superior Aptiano-Albiano $\left(\mathrm{K}_{2}^{\mathrm{ap}-\mathrm{al}}\right)$.

Yaciendo en forma discordante sobre esta corteza oceánica, aparece la formación vulcanógenosedimentaria, del Cretácico Superior Albiano - Campaniano Inferior $\left(\mathrm{K}_{2}{ }^{\text {al-cp }}{ }_{1}\right)$. La potencia de dicha formación aumenta hacia el Sur alcanzando los 3,000 metros. La parte superior está compuesta por tobas y brechas de lavas mayormente basálticas y andesíticas afectadas por cataclasis. También aparecen lavas dacíticas y riolíticas, si bien en menor escala. En la parte media de la formación, las lavas presentan estructuras porfíricas, presentando poros y amigdalas en la base.

En contacto discordante sobre estas lavas yace la formación areno-arcilloso-carbonatada de la región nerítica interna, de edad Cretácico Superior Campaniano Superior al Eoceno Medio parte Inferior $\left(\mathrm{K}_{2}{ }^{\mathrm{cp}}{ }_{2}-\mathrm{P}_{2}{ }^{1}\right)$, con potencias que varían desde las primeras centenas de metros hasta los 2,500 metros. La formación está compuesta por secuencias terrígenas, arcillosas y carbonatadas.

También en forma discordante, aparece la formación areno-carbonatado-arcillosa de la región nerítica externa, de edad Eoceno Medio parte Superior al Quaternario Reciente $\left(\mathrm{P}_{2}{ }^{3}-\mathrm{Q}\right)$ con espesores que varían de 50 a 1,800 metros. Predominan aquí las aleurolitas polimícticas, las arcillas arenosas, las gravelitas polimícticas, las rocas efusivas, las calizas organógenas, las margas arenosas y las calizas fragmentarias, así como arcillas calcáreas semi-consolidadas.

\section{Apuntes a la geología del área}

Se usó una red de 25 x 10 metros para el muestreo de suelos en el sector de estudios. Para los datos de elevación se consideró como "cero" el nivel del arroyo. La red fue trazada a brújula y cinta métrica. Las muestras fueron tomadas a $0.2-0.3$ metros de profundidad, con un peso inicial de 200 - 250 gramos. Cada muestra fue secada al Sol, desmenuzada y tamizada a $-1 \mathrm{~mm}$ y enviada 
al laboratorio, donde fueron pulverizadas a 200 Mesh y sometidas a análisis espectral semicuantitativo en cabina de dispersión para 35 elementos con un $100 \%$ de repetición.

Durante los muestreos fueron utilizadas fichas codificadas (Valls, 1993). La información recogida en las fichas permite caracterizar la zona muestreada como un eluvio pardo oscuro con fragmentos menores de 2-3 centímetros de tobas muy alteradas. El eluvio fué tomado del horizonte B del suelo y se presenta generalmente hematizado. Por toda el área muestreada existen pequeños afloramientos intemperizados de tobas. Dada la suavidad de las pendientes, es de esperar que el eluvio se encuentre poco transportado. Es interesante señalar la ausencia de todo tipo de vegetación en los alrededores del punto $2 / 8$, a pesar de la existencia de una delgada capa de suelo correspondiente al horizonte A-B.

El corte artificial existente entre los perfiles "0" y "2", permite una completa descripción de la litología del Sector. Tomando como punto de partida la caída de agua al Sur del área, podemos observar el corte geológico exhibido en la Fig. 1.

Leyenda

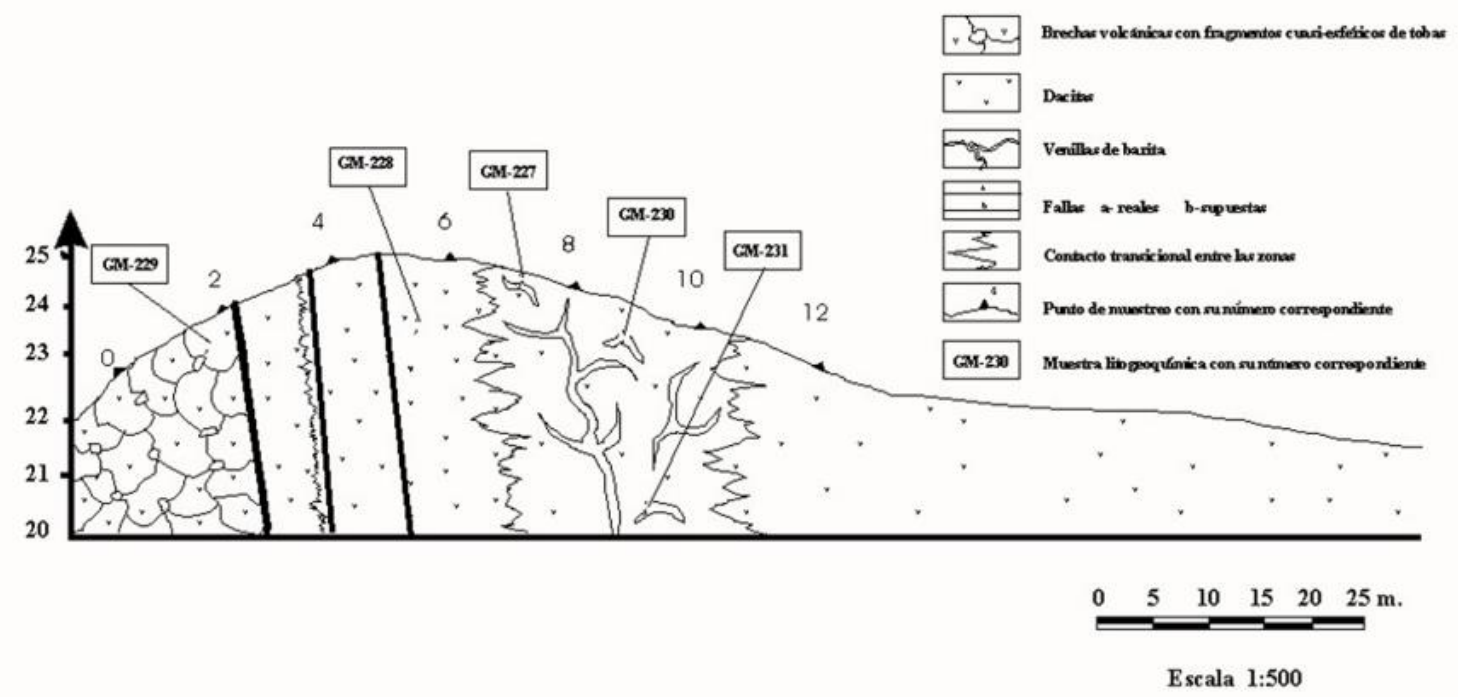

Figura 1.Diagrama del corte geológico del Sector "Los Tramojos" a través del perfil 2.

Los primeros 17 metros están conformados por brechas volcánicas intermedias a ácidas, con gran abundancia de fragmentos ligeramente angulares, con diámetros por lo general inferiores a los 20 centímetros.

De los 17 a los 22 metros se observan tobas intermedias, presumiblemente dacitas, de color pardo claro, con huellas de una limonitización incipiente. La roca es en general deleznable, observándose evidencias de un contacto de tipo tectónico con las brechas descritas con anterioridad.

De los 22 a los 40 metros se mantiene la presencia de las mismas tobas, pero en esta ocasión muy alteradas, con limonitización, hematización, zeolitización y cloritización. Se notan algunas fallas paralelas y cierto alineamiento en la roca. Dos de las fallas mejor expuestas se encuentran situadas a los 23 y 30 metros respectivamente y sus condiciones de yacencia son $285 / 75^{\circ}$. Es de señalar que la primera de estas fallas se encuentra fuertemente hematizada y limonitizada, en cambio que en la segunda predomina el proceso de zeolitización. 
De los 40 a los 60 metros aflora el cuerpo mineral principal, compuesto por pirita, magnetita, venillas de barita, así como apatito, esfalerita, sulfuros de cobre y galenita. Debido a la presencia de estos minerales, las tobas adquieren un color muy oscuro y son muy pesadas (Fig. 2).

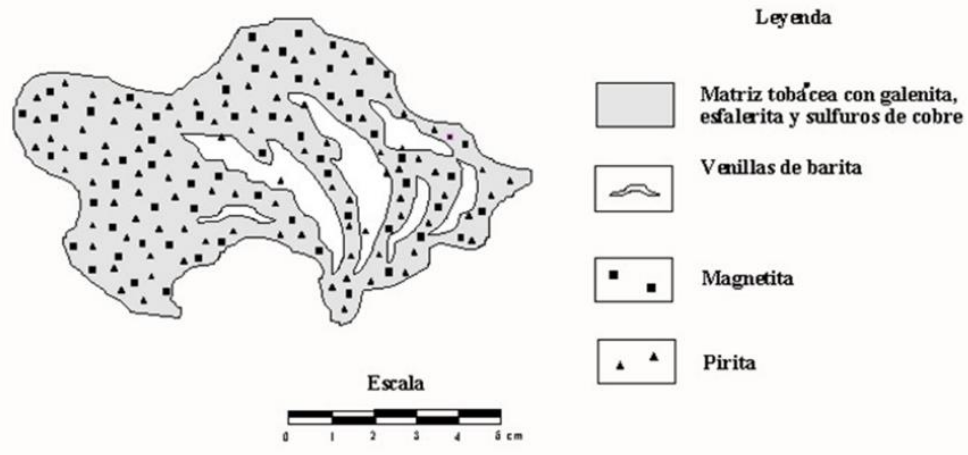

\begin{tabular}{|l|c|c|c|c|c|c|c|c|c|c|c|c|c|c|}
\hline [p.p.m.] & As & $\mathrm{P}^{2}$ & $\mathrm{~Pb}$ & $\mathrm{Sn}$ & $\mathrm{Ag}$ & $\mathrm{Ni}$ & $\mathrm{Mo}$ & $\mathrm{Cu}$ & $\mathrm{Co}$ & $\mathrm{Zn}$ & $\mathrm{Ga}$ & $\mathrm{Mn}$ & $\mathrm{V}$ & $\mathrm{Ba}$ \\
\hline GM-230 & 300 & 400 & 1 & 2 & 2 & 10 & 200 & 300 & 40 & 40 & 10 & 400 & 80 & 600 \\
\hline
\end{tabular}

Figura 2. Estructura y composición de la muestra GM-230 del sector "Los Tramojos".

El resto del perfil hasta los 115 metros lo componen las mismas tobas descritas en el intervalo de 17 a 22 metros. El contacto entre las zonas descritas es de carácter transicional y paulatino.

La documentación "in situ" de las principales alteraciones observadas en el corte permitió la confección de un corte epigenético del Sector (Fig. 3).

\section{Leyenda}

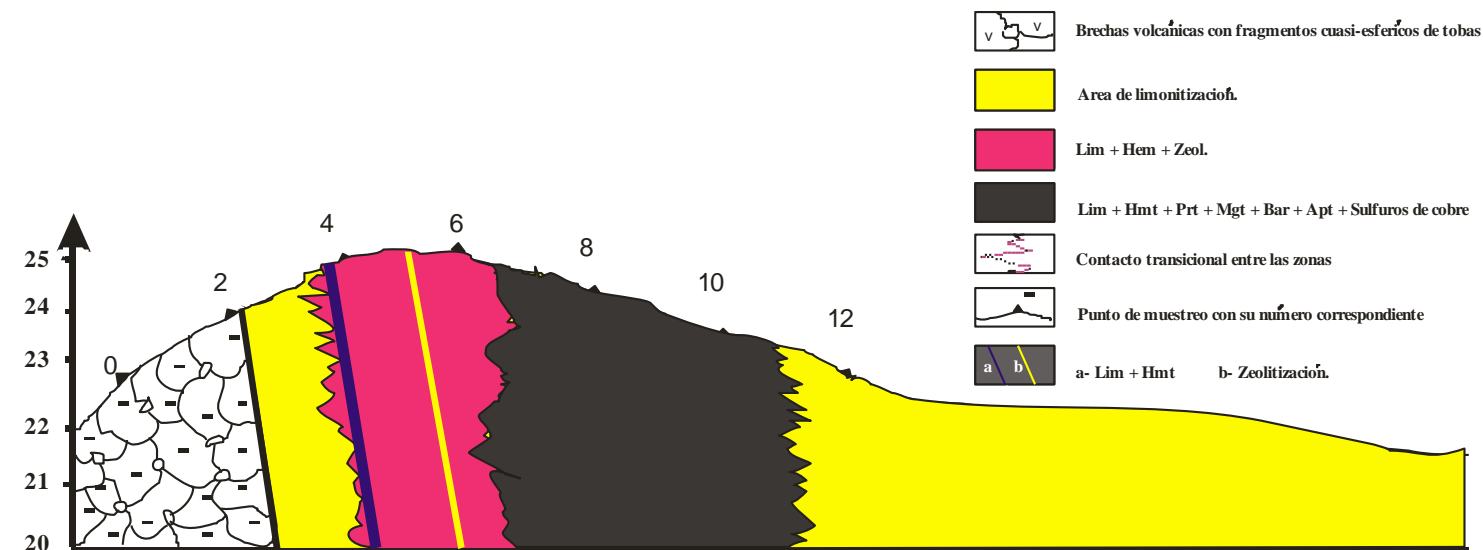

$\begin{array}{lllllll}0 & 5 & 10 & 15 & 20 & 25 & \mathrm{~m}\end{array}$

Escala 1:500

Figura 3. Diagrama del corte epigenético del sector "Los Tramojos" a través del perfil 2.

En el mismo podemos apreciar la existencia de una zona mineral, acompañada de un frente de alteración y tectonizacíon, rodeada a su vez por tobas poco alteradas en contacto lateral con una 
zona de brechas volcánicas al inicio del corte. La presencia de concentraciones relativamente elevadas de hierro, cobre y cinc, las condiciones de arco insular de la región, así como la secuencias litológicas expuestas en las figuras 2 y 3 y explicadas en el texto, permiten suponer que el depósito mineral estudiado corresponde al tipo Kuroko de sulfuros masivos y de génesis vulcano-exhalativa submarina.

Es esta la primera vez que se presenta un estudio geoquímico de un yacimiento de este tipo en Cuba, si bien no es la primera vez que se menciona la posibilidad de la existencia de yacimientos de tipo Bechee o Kuroko en dicho País (Humberto Alvarez, comun. pers.).

\section{Estudio geoquímico del área}

\section{Modelaje geoquímico propuesto}

La tabla 1 presenta la información inicial con que se contaba. Un total de 11 elementos y 21 muestras, para un total de 231 datos. Teniendo en cuenta lo limitado de la información, se trabajó con la totalidad de la población.

Tabla 1. Datos reales del sector "Los Tramojos". Todos los datos se expresan en p.p.m.

\begin{tabular}{lrrrrrrrrrrr} 
Muestra & P & Pb & Sn & \multicolumn{1}{c}{ Ag } & Ni & Cu & Co & Zn & Mn & V & Ga \\
\hline N 4/0 & 30 & 2 & 1 & 0.08 & 100 & 80 & 40 & 100 & 1000 & 100 & 20 \\
N 4/2 & 30 & 2 & 1 & 0.08 & 200 & 100 & 60 & 100 & 1000 & 100 & 20 \\
N 4/4 & 100 & 4 & 2 & 0.1 & 20 & 80 & 10 & 100 & 800 & 100 & 20 \\
N 4/6 & 100 & 1 & 2 & 0.1 & 30 & 100 & 10 & 100 & 1000 & 100 & 20 \\
N 4/8 & 200 & 3 & 2 & 0.1 & 30 & 100 & 10 & 200 & 1000 & 100 & 20 \\
N 4/10 & 80 & 2 & 2 & 0.1 & 40 & 100 & 20 & 200 & 1000 & 100 & 20 \\
N 4/12 & 100 & 3 & 2 & 0.1 & 60 & 100 & 30 & 200 & 1000 & 100 & 20 \\
N 2/0 & 80 & 2 & 2 & 0.08 & 30 & 100 & 20 & 200 & 800 & 100 \\
N 2/2 & 80 & 2 & 2 & 0.08 & 10 & 100 & 10 & 200 & 800 & 100 \\
N 2/4 & 80 & 2 & 2 & 0.08 & 20 & 80 & 10 & 200 & 600 & 80 \\
N 2/6 & 100 & 1 & 1 & 0.08 & 8 & 80 & 8 & 100 & 400 & 80 \\
N 2/8 & 60 & 2 & 1 & 0.08 & 10 & 80 & 10 & 200 & 600 & 80 \\
N 2/10 & 80 & 3 & 1 & 0.1 & 30 & 100 & 30 & 200 & 800 & 100 & 20 \\
N 2/12 & 60 & 2 & 1 & 0.1 & 30 & 80 & 10 & 200 & 600 & 80 \\
N 0/0 & 100 & 1 & 1 & 0.08 & 6 & 60 & 4 & 100 & 200 & 60 \\
N 0/2 & 100 & 1 & 1 & 0.08 & 4 & 60 & 4 & 100 & 200 & 60 \\
N 0/4 & 100 & 1 & 1 & 0.08 & 6 & 80 & 6 & 100 & 200 & 80 \\
N 0/6 & 80 & 1 & 1 & 0.08 & 6 & 60 & 6 & 100 & 200 & 80 \\
N 0/8 & 80 & 2 & 2 & 0.2 & 6 & 80 & 8 & 200 & 300 & 80 \\
N 0/10 & 80 & 1 & 1 & 0.08 & 6 & 60 & 6 & 100 & 100 & 80 \\
N 0/12 & 80 & 1 & 2 & 0.08 & 10 & 100 & 20 & 100 & 800 & 80 \\
\hline
\end{tabular}

Fuente: Laboratorio espectral de la Empresa de Geología de Santa Clara, Cuba.

Las figuras 4 - 6, muestran el comportamiento individual de los elementos analizados. 


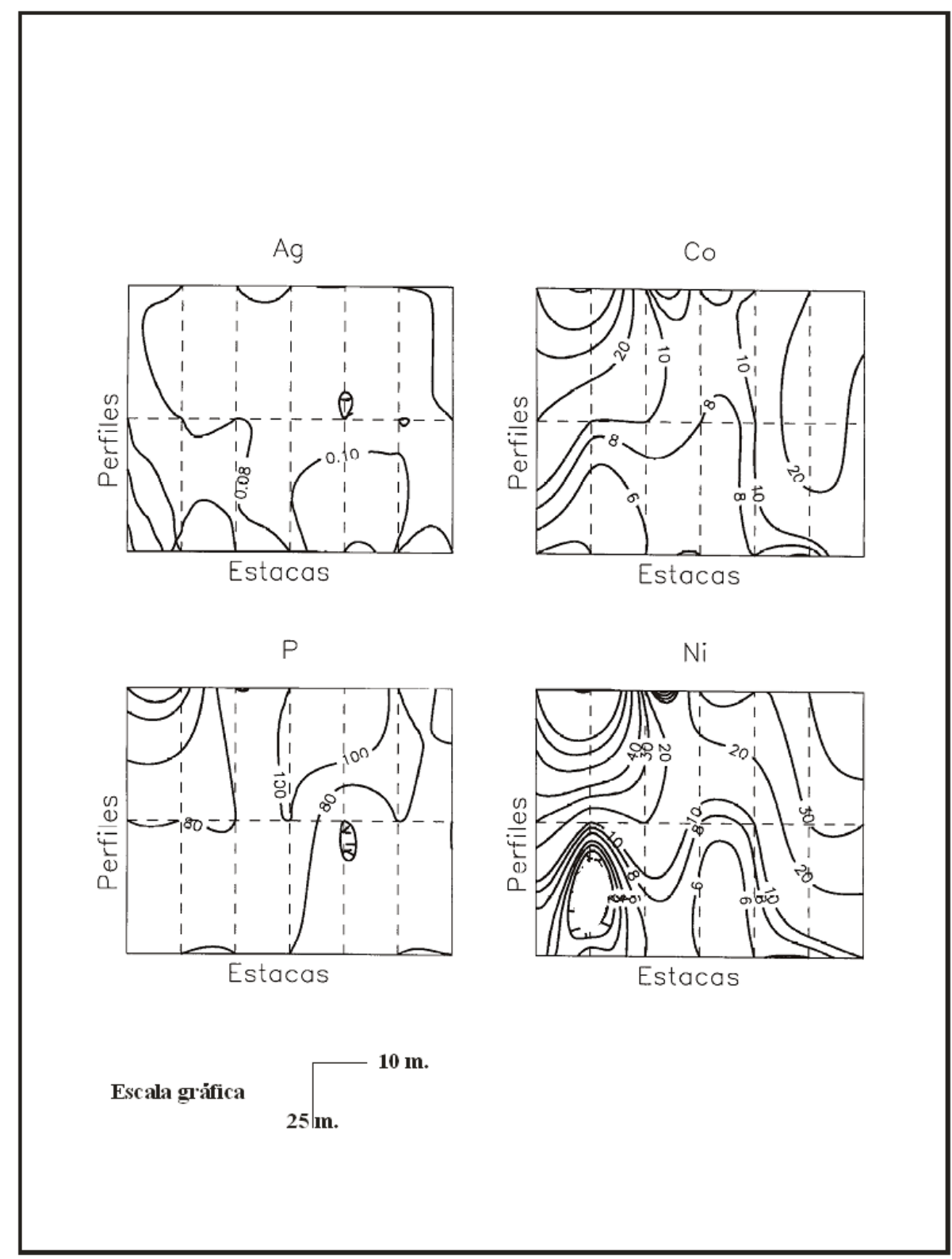

Figura 4. Mapas monoelementales del cobalto, niquel, fósforo y plata en el sector "Los Tramojos". 


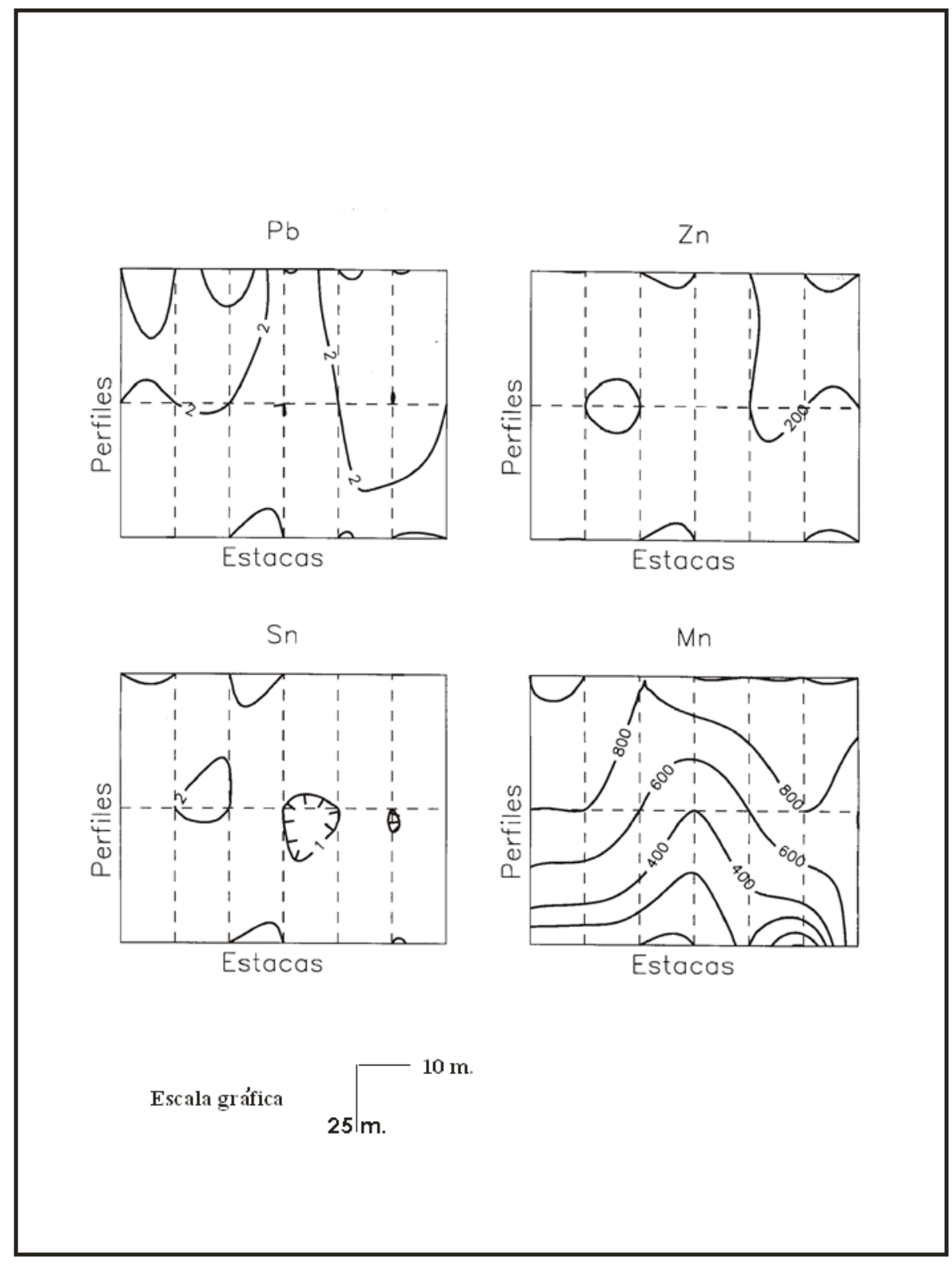

Figura 5. Mapas monoelementales del plomo, cinc, estaño y manganeso en el sector "Los Tramojos". 


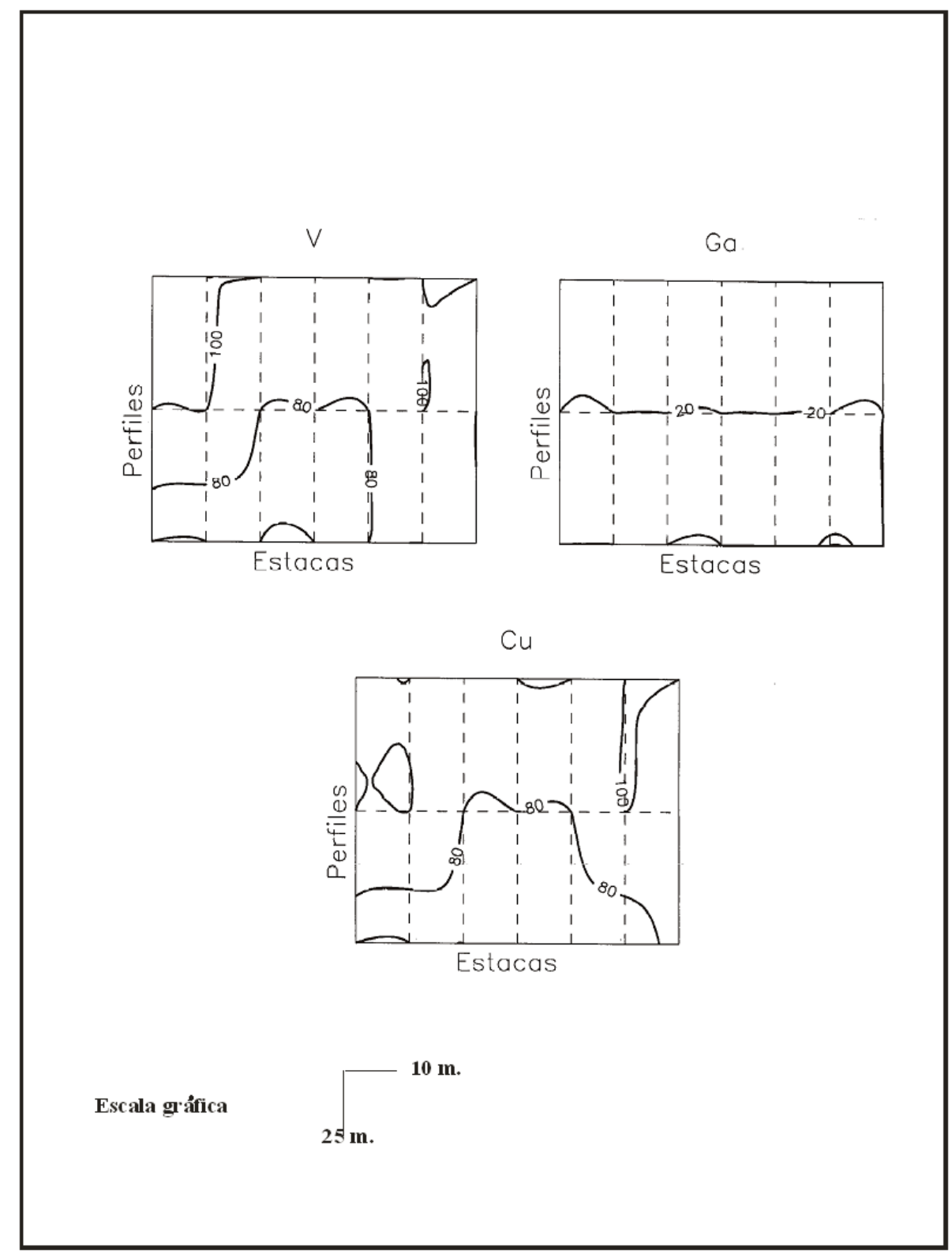

Figura 6. Mapas monoelementales del vanadio, galio y cobre en el sector "Los Tramojos".

Dichos mapas fueron obtenidos inicialmente mediante el uso del software SURFER a partir de una red de 61 x 25 unidades, y con un grado elevado de suavización en las curvas. Como puede apreciarse, el estudio individual de dichos elementos no nos brinda una información sensiblemente útil. Esto definió que el curso del modelaje geomatemático de dichos datos siguiera la secuencia siguiente.

\section{Principales parámetros estadísticos}

El software SYSTAT permitió obtener los valores de los principales parámetros estadísticos de la población, incluyendo la determinación de las Leyes de Distribución de cada elemento por el método de la Asimetría y del Exceso. De esta forma quedó establecido que el níquel y el fósforo respondían a una distribución log normal, en tanto que la plata no respondía a ninguna de las dos leyes, razón por la cual se asumió la misma como log normal. 
La tabla 2 muestra un resumen de los análisis estadísticos efectuados. La misma incluye además la determinación de los niveles de anomalía (1), el coeficiente de concentración (2), y el grado de contraste de las anomalías (3) y (4).

Tabla 2. Resumen del estudio estadístico de los datos del sector "Los Tramojos". Total de observaciones: 21.

\begin{tabular}{|c|c|c|c|c|c|c|c|c|c|c|c|}
\hline Par添etro & Ag (log) & Co & $\mathbf{C u}$ & $\mathbf{G a}$ & Mn & $\mathrm{Ni}(\log )$ & P (log) & $\mathbf{P b}$ & Sn & $\mathbf{V}$ & $\mathbf{Z n}$ \\
\hline Valor máximo & -0.7 & 60.0 & 100.0 & 20.0 & 1000.0 & 2.3 & 2.3 & 4.0 & 2.0 & 100.0 & 200.0 \\
\hline Valor mínimo & -1.1 & 4.0 & 60.0 & 8.0 & 100.0 & 0.6 & 1.5 & 1.0 & 1.0 & 60.0 & 100.0 \\
\hline Valor medio & -1.1 & 15.8 & 84.8 & 17.5 & 638.1 & 1.2 & 1.9 & 1.9 & 1.5 & 87.6 & 147.6 \\
\hline Varianza & 0.0 & 196.0 & 236.2 & 20.8 & 107476.2 & 0.2 & 0.0 & 0.7 & 0.3 & 179.1 & 2619.1 \\
\hline Desviación standard & 0.1 & 14.0 & 15.4 & 4.6 & 327.8 & 0.5 & 0.2 & 0.9 & 0.5 & 13.4 & 51.2 \\
\hline Error standard & 0.0 & 3.1 & 3.4 & 1.0 & 71.5 & 0.1 & 0.0 & 0.2 & 0.1 & 2.9 & 11.2 \\
\hline Asimetría & 10.3 & 4.1 & -1.1 & -0.1 & -1.4 & -0.2 & 3.0 & 0.4 & -2.2 & -0.5 & -2.2 \\
\hline Exceso & 2.9 & 2.0 & -0.5 & -1.4 & -0.4 & 0.6 & -0.9 & 0.8 & 0.1 & -0.6 & 0.1 \\
\hline Sumatoria & -22.0 & 332.0 & 1780.0 & 368.0 & 13400.0 & 26.1 & 39.9 & 39.0 & 31.0 & 1840.0 & 3100.0 \\
\hline Moda absoluta & -1.1 & 10.0 & 100.0 & 20.0 & 1000.0 & 1.5 & 1.9 & 2.0 & 1.0 & 100.0 & 100.0 \\
\hline Mediana & -1.1 & 10.0 & 80.0 & 20.0 & 800.0 & 1.3 & 1.9 & 2.0 & 1.0 & 80.0 & 100.0 \\
\hline Contraste & 3.5 & 3.1 & 1.1 & 0.7 & 1.5 & 3.3 & 2.7 & 1.3 & 1.1 & 1.3 & 3.4 \\
\hline Coef. de concentración & 1.8 & 2.3 & 8.0 & 0.6 & 0.8 & 30.2 & 0.1 & 1.1 & 2.9 & 2.2 & 0.5 \\
\hline$A+3$ & -0.8 & 57.8 & 130.9 & 31.2 & 1621.6 & 2.6 & 2.4 & 4.4 & 3.0 & 127.8 & 301.2 \\
\hline $\mathrm{A}+2$ & -0.9 & 43.8 & 115.5 & 26.6 & 1293.8 & 2.2 & 2.3 & 3.6 & 2.5 & 114.4 & 250.0 \\
\hline $\mathrm{A}+1$ & -1.0 & 29.8 & 100.1 & 22.1 & 965.9 & 1.7 & 2.1 & 2.7 & 2.0 & 101.0 & 198.8 \\
\hline Fondo & -1.1 & 15.8 & 84.8 & 17.5 & 638.1 & 1.2 & 1.9 & 1.9 & 1.5 & 87.6 & 147.6 \\
\hline A-1 & -1.1 & 1.8 & 69.4 & 13.0 & 310.3 & 0.8 & 1.7 & 1.0 & 1.0 & 74.2 & 96.4 \\
\hline A-2 & -1.2 & -12.2 & 54.0 & 8.4 & -17.6 & 0.3 & 1.6 & 0.2 & 0.5 & 60.9 & 45.3 \\
\hline A-3 & -1.3 & -26.2 & 38.7 & 3.9 & -345.4 & -0.1 & 1.4 & -0.7 & -0.1 & 47.5 & -5.9 \\
\hline Rango & 0.4 & 56.0 & 40.0 & 12.0 & 900.0 & 1.7 & 0.8 & 3.0 & 1.0 & 40.0 & 100.0 \\
\hline
\end{tabular}

Ecuación (1) $X_{\text {anom }}=M x \pm n * \sigma$

donde,

$X_{\text {anom }}$ - valor anómalo

Mx - valor medio

$\sigma \quad$ - desviación estándar

$n \quad$ - niveles de anomalía $(\mathrm{n}=1,2,3)$

Ecuación (2) $K_{c_{i}}=M x_{i} * \frac{1}{\text { Clark }_{i}}$

donde,

Kci - coeficiente de concentración

Mx - valor medio

Clark $_{i}$ - Clark de cada elemento

Ecuación (3) $\varepsilon=\sqrt[3]{\frac{(M x+3 * \sigma)}{M x}}$

donde,

$\varepsilon \quad$ - múltiplo estándar 
Mx - valor medio

$\sigma \quad$ - desviación estándar

Ecuación $4 \gamma=\frac{1}{\log \varepsilon} * \log \left(\frac{X \max }{M x}\right)$

donde,

$\tau \quad$ - grado de contraste de las anomalías

$\varepsilon \quad$ - múltiplo estándar

$M x \quad$ - valor medio

Xmax - valor máximo

Los Clarke del fósforo, manganeso, cobalto, níquel, cobre, cinc, estaño y plomo, fueron tomados -o estimados- en base a los datos brindados por K. H. Wedepohl (1978, TII-2 - TII-5), en tanto que los Clarke del vanadio, la plata y el galio se tomaron según K.K.Turekian y K.H. Wedepohl K.H. (Rostes y Lange, 1972, 230).

La Fig. 7 muestra los resultados del análisis del Clark-concentración. En el mismo no se representó el fósforo, pues es posible que su gran abundancia en la capa vegetal se deba a factores externos (abono del campo) y no a la presencia de apatito u otros minerales contenedores de dicho elemento, como sí sucede en las muestras litogeoquímicas. La presencia del cinc entre los elementos que se concentran confirma la teoría de la relación entre dicho mineral y el cobre, en el sentido de que el cobre siempre aparece asociado al cinc y nunca sólo (Evans, 1987, 206).

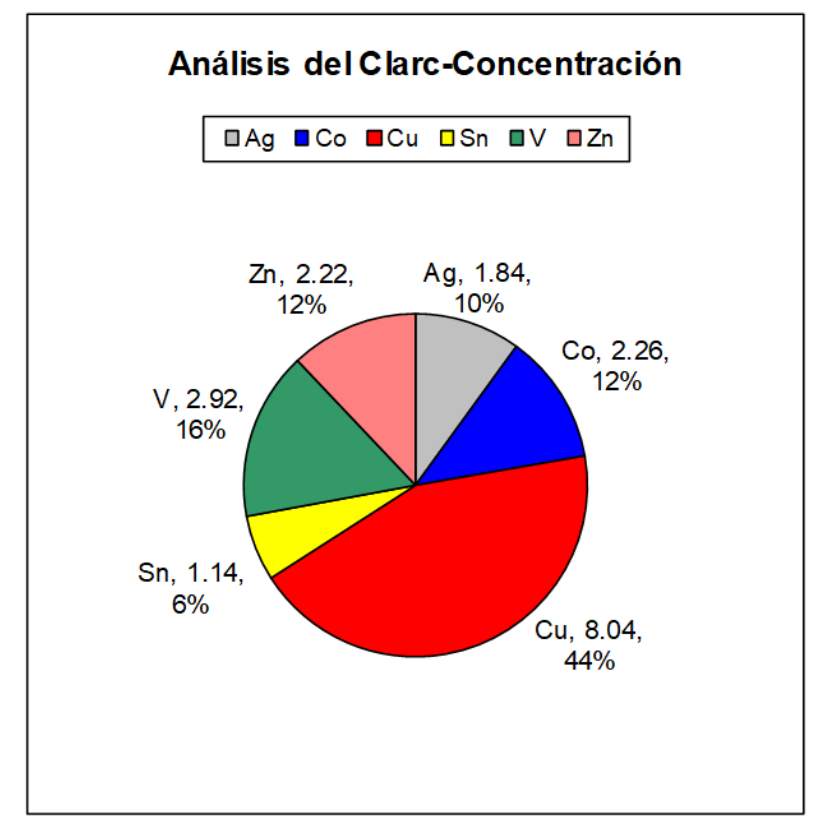

Figura 7. El método de los Clark-concentraciones muestra claramente cuáles elementos se concentran en las muestras de suelo del Sector "Los Tramojos".

La ausencia de plomo -según la misma fuente- implica la ausencia de riolitas en el corte, lo cual si bien no es característico para los yacimientos del tipo Kuroko, no resulta una limitante para así ser considerado, según Denis P. Cox y Donald A. Singer (1986, 189 y ss.). 


\section{Coeficientes correlacionales}

En 1989, buscando un método efectivo para el mapeo de cuerpos talcosos en rocas del complejo metamórfico del Escambray (Valls y Nuñez, 1989, 12 y ss), utilicé la representación gráfica de los análisis correlacionales binarios de formaciones geológicas independientes, para determinar no sólo los elementos que las caracterizaban, sino también la relación que mantenían entre ellos, determinando de esa forma los coeficientes correlacionales (C.C.) típicos para cada formación.

El siguiente paso consistía en comparar todos los C.C. obtenidos, eliminando aquellos que tuvieran comportamientos similares en distintas formaciones geológicas. El resultado final fue un grupo de C.C. cuyos valores extremos mapeaban exclusivamente la formación geológica para la cual fueron determinados. Posteriormente he tenido la oportunidad de comprobar la eficiencia de la técnica de los C.C. en variadas situaciones geológicas, razón por la cual fue empleada en este estudio.

La Tabla 3 fue obtenida por medio del software SYSTAT y muestra los resultados del análisis de correlación binario por el método de Pearson para la totalidad de las muestras del Sector "Los Tramojos".

Tabla 3. Resultados del análisis de correlación y varianza (en rojo) en el sector "Los Tramojos". Total de observaciones: 21.

\begin{tabular}{cccccccccccc} 
& $\mathbf{A g}(\log )$ & $\mathbf{C o}$ & $\mathbf{C u}$ & $\mathbf{G a}$ & $\mathbf{M n}$ & $\mathbf{N i}(\log )$ & $\mathbf{P}(\log )$ & $\mathbf{P b}$ & $\mathbf{S n}$ & $\mathbf{V}$ & $\mathbf{Z n}$ \\
\hline $\mathbf{A g}(\log )$ & & & & & & & & & $\mathbf{4 . 6 0}$ & $\mathbf{4 . 5 0}$ & \\
$\mathbf{C o}$ & & & $\mathbf{4 . 4 0}$ & $\mathbf{4 . 5 0}$ & $\mathbf{4 . 2 0}$ & $\mathbf{4 . 0 0}$ & $\mathbf{5 . 8 0}$ & & & $\mathbf{4 . 3 0}$ \\
$\mathbf{C u}$ & & 0.52 & & $\mathbf{4 . 1 0}$ & $\mathbf{4 . 0 0}$ & $\mathbf{4 . 3 0}$ & & $\mathbf{4 . 5 0}$ & $\mathbf{4 . 3 0}$ & $\mathbf{4 . 1 0}$ & $\mathbf{4 . 5 0}$ \\
$\mathbf{G a}$ & & 0.43 & 0.78 & & $\mathbf{4 . 1 0}$ & $\mathbf{4 . 3 0}$ & & $\mathbf{4 . 3 0}$ & $\mathbf{4 . 4 0}$ & $\mathbf{4 . 2 0}$ \\
$\mathbf{M n}$ & & 0.64 & 0.86 & 0.80 & & $\mathbf{4 . 0 0}$ & & $\mathbf{4 . 3 0}$ & $\mathbf{4 . 4 0}$ & $\mathbf{4 . 0 0}$ & $\mathbf{4 . 6 0}$ \\
$\mathbf{N i}(\log )$ & & 0.85 & 0.63 & 0.62 & 0.85 & & $\mathbf{5 . 6 0}$ & $\mathbf{4 . 4 0}$ & & $\mathbf{4 . 1 0}$ \\
$\mathbf{P}(\log )$ & & -0.69 & & & & & -0.49 & & & $\mathbf{4 . 5 0}$ & \\
$\mathbf{P b}$ & 0.35 & & 0.44 & 0.57 & 0.57 & 0.51 & & & $\mathbf{4 . 5 0}$ & $\mathbf{4 . 3 0}$ & $\mathbf{4 . 4 0}$ \\
$\mathbf{S n}$ & 0.39 & & 0.59 & 0.53 & 0.51 & & 0.39 & 0.39 & & $\mathbf{4 . 5 0}$ & $\mathbf{4 . 5 0}$ \\
$\mathbf{V}$ & & 0.56 & 0.79 & 0.65 & 0.84 & 0.75 & & 0.63 & 0.47 & & \\
$\mathbf{Z n}$ & 0.39 & & 0.46 & 0.53 & 0.33 & & & 0.51 & 0.43 & \\
\hline
\end{tabular}

Nota: De acuerdo con el criterio de Student, sólo son sensibles las correlaciones mayores que 0,332 y menores que $-0,332$.

Las correlaciones sensibles fueron determinadas por medio del criterio de Student (5) asumiendo $\mathrm{t}_{\mathrm{cr}}=2$ (Kazhdan, 1979).

Ecuación (5) $t_{c r}=\frac{\sqrt{n-1}}{\sqrt{1-r^{2}}}$

donde,

$t_{c r} \quad$ - valor crítico de la distribución de Student

$r \quad$ - coeficiente de correlación

n - cantidad de muestras 
Para la representación gráfica del análisis de correlación binario, se ordenan primeramente las correlaciones sensibles en orden descendente y luego, por medio de la ecuación (6), se calcula el valor en centímetros de cada para correlacional. Dicho valor es la "distancia" a que se encuentran cada elemento en el espacio. Los resultados de dicho procedimiento se muestran en la Tabla 4.

Tabla 4. Distancia correlacional entre los elementos analizados. No se incluyen las correlaciones no sensibles.

\begin{tabular}{lrlllcc} 
Correlación & \multicolumn{1}{c}{ r } & $\mathbf{L}$ & & Correlación & $\mathbf{r}$ & $\mathbf{L}$ \\
\hline $\mathrm{Cu}: \mathrm{Mn}$ & 0.86 & 4.00 & & $\mathrm{Ga}: \mathrm{Sn}$ & 0.53 & 4.40 \\
$\mathrm{Co}: \mathrm{Ni}$ & 0.85 & 4.00 & & $\mathrm{Co}: \mathrm{Cu}$ & 0.52 & 4.40 \\
$\mathrm{Mn}: \mathrm{Ni}$ & 0.85 & 4.00 & & $\mathrm{Mn}: \mathrm{Sn}$ & 0.51 & 4.40 \\
$\mathrm{Mn}: \mathrm{V}$ & 0.84 & 4.00 & & $\mathrm{Ni}: \mathrm{Pb}$ & 0.51 & 4.40 \\
$\mathrm{Mn}: \mathrm{Ga}$ & 0.8 & 4.10 & & $\mathrm{~Pb}: \mathrm{Zn}$ & 0.51 & 4.40 \\
$\mathrm{Cu}: \mathrm{V}$ & 0.79 & 4.10 & & $\mathrm{Sn}: \mathrm{V}$ & 0.47 & 4.50 \\
$\mathrm{Cu}: \mathrm{Ga}$ & 0.78 & 4.10 & & $\mathrm{Cu}: \mathrm{Zn}$ & 0.46 & 4.50 \\
$\mathrm{Ni}: \mathrm{V}$ & 0.75 & 4.10 & & $\mathrm{Cu}: \mathrm{Pb}$ & 0.44 & 4.50 \\
$\mathrm{Ga}: \mathrm{V}$ & 0.65 & 4.20 & & $\mathrm{Co}: \mathrm{Ga}$ & 0.43 & 4.50 \\
$\mathrm{Co}: \mathrm{Mn}$ & 0.64 & 4.20 & & $\mathrm{Sn}: \mathrm{Zn}$ & 0.43 & 4.50 \\
$\mathrm{Ni}: \mathrm{Cu}$ & 0.63 & 4.30 & & $\mathrm{Ag}: \mathrm{Sn}$ & 0.39 & 4.50 \\
$\mathrm{~Pb}: \mathrm{V}$ & 0.63 & 4.30 & & $\mathrm{Ag}: \mathrm{Zn}$ & 0.39 & 4.50 \\
$\mathrm{Ni}: \mathrm{Ga}$ & 0.62 & 4.30 & & $\mathrm{~Pb}: \mathrm{Sn}$ & 0.39 & 4.50 \\
$\mathrm{Cu}: \mathrm{Sn}$ & 0.59 & 4.30 & & $\mathrm{P}: \mathrm{Sn}$ & 0.39 & 4.50 \\
$\mathrm{Mn}: \mathrm{Pb}$ & 0.57 & 4.30 & & $\mathrm{Ag}: \mathrm{Pb}$ & 0.35 & 4.60 \\
$\mathrm{Ga}: \mathrm{Pb}$ & 0.57 & 4.30 & & $\mathrm{Mn}: \mathrm{Zn}$ & 0.33 & 4.60 \\
$\mathrm{Co}: \mathrm{V}$ & 0.56 & 4.30 & & $\mathrm{P}: \mathrm{Ni}$ & -0.49 & 5.60 \\
$\mathrm{P}: \mathrm{Co}$ & -0.69 & 5.80 & & & \\
\hline
\end{tabular}

Ecuación (6) $L=4 c m+\left(1-\frac{r_{i}}{r_{\max }}\right)$

donde,

L $\quad$ - distancia entre los parámetros del par correlacional

$r_{\max } \quad$ - valor máximo de las correlaciones

$r \quad$ - valor de correlación del par correlacional

La explicación detallada del método de los C.C. cae fuera del objetivo de este trabajo. El interesado puede consultar al respecto el artículo antes citado (Valls y Nuñez, 1989).

La figura 8 muestra el diagrama correlacional del Sector "Los Tramojos", así como la propuesta de dos C.C. 


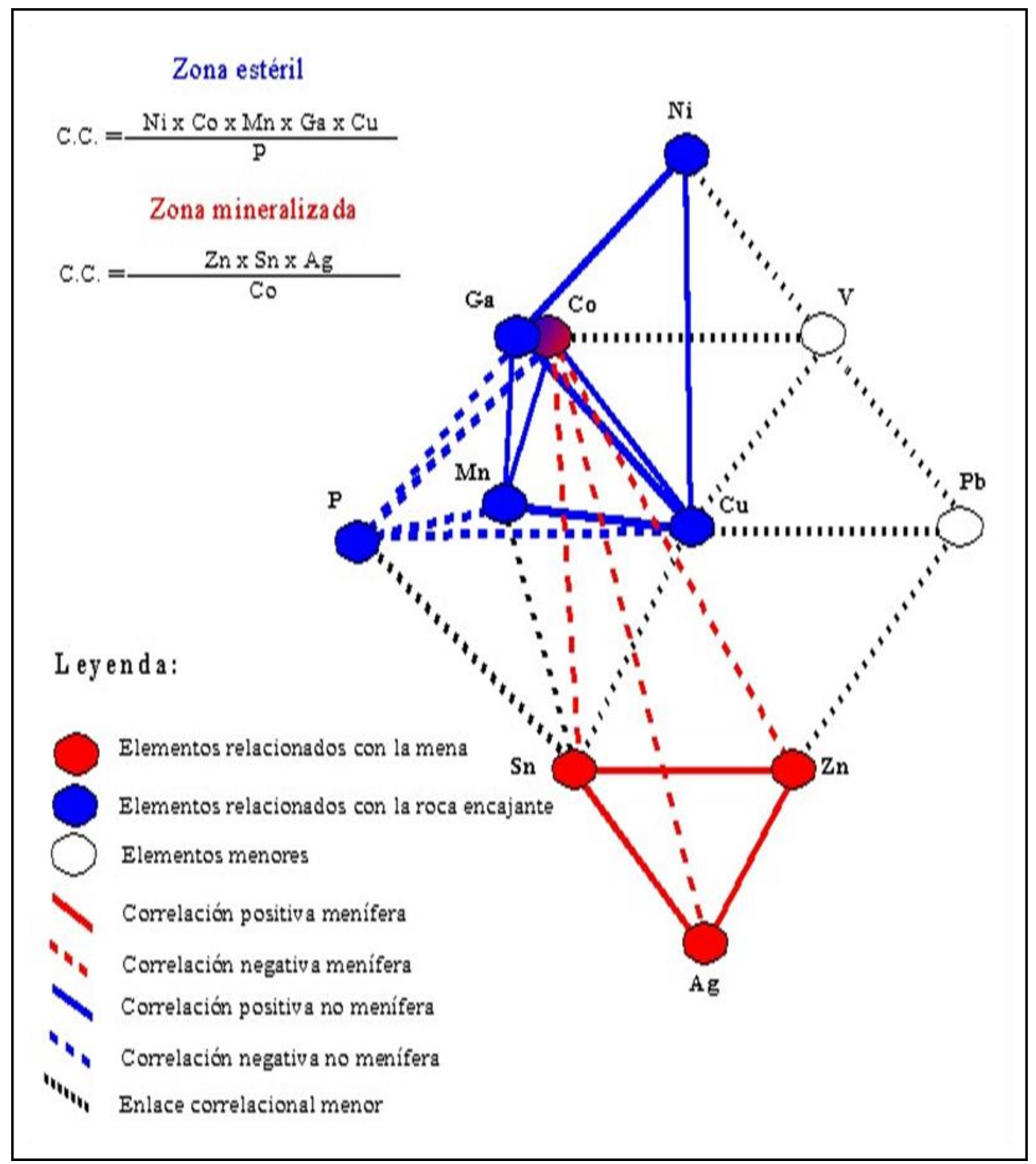

Figura 8. Diagrama correlacional del sector "Los Tramojos", con sus coeficientes correlacionales característicos.

El primero (Fig. 9), corresponde mayormente al área de desarrollo de las brechas volcánicas (zona estéril), en tanto que el segundo (Figs. 10-11), corresponde al área de desarrollo de la zona mineralizada incluyendo en ella el área de las "menas negras" y el área de desarrollo de la zona de alteración. 


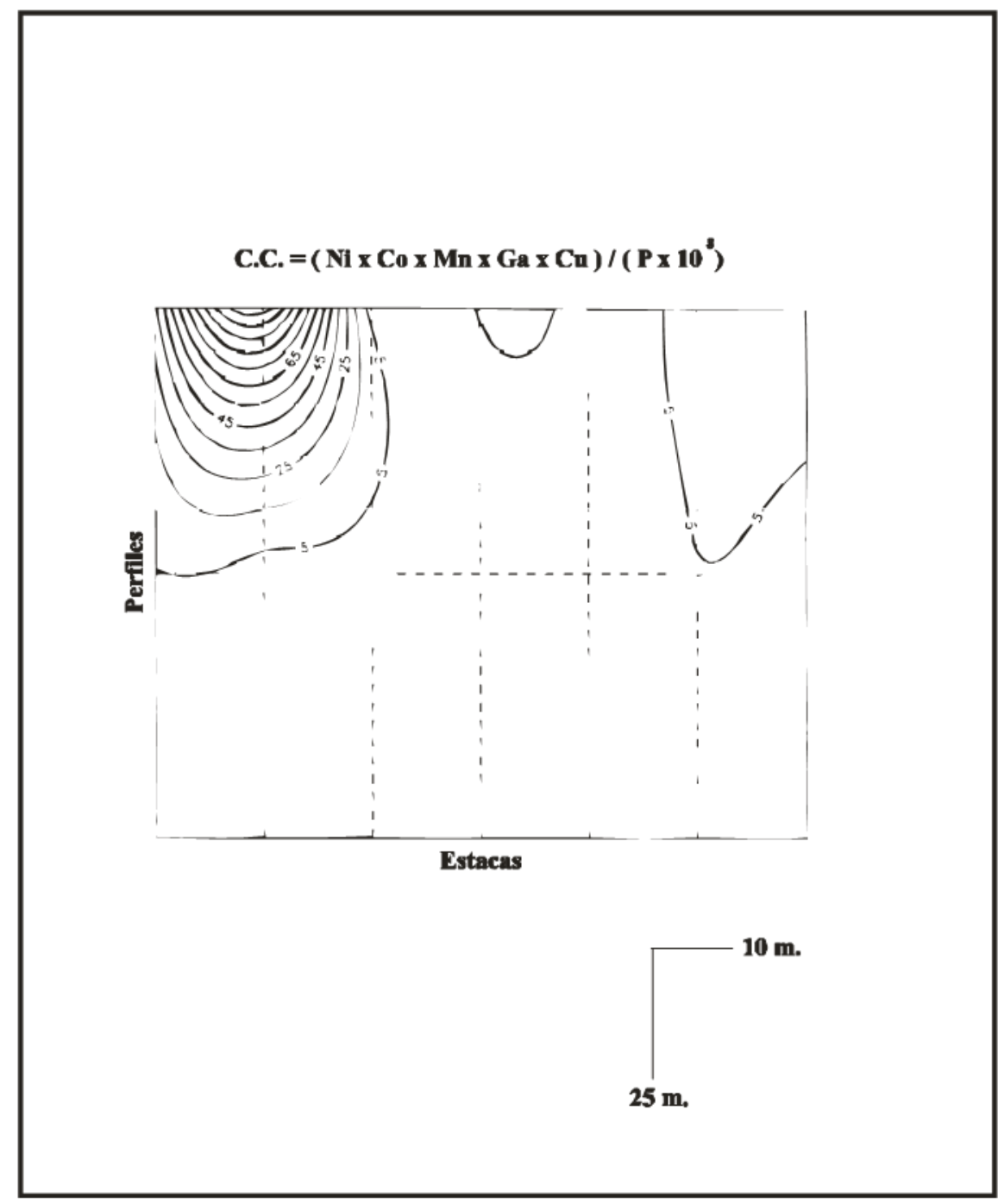

Figura 9. El C.C. (Ni $x$ Co $\times$ Mn $\times$ Ga $\times$ Cu) /(P x 108) permite el mapeo de la zona estéril en el sector "Los Tramojos", representada mayormente por las brechas volcánicas. 


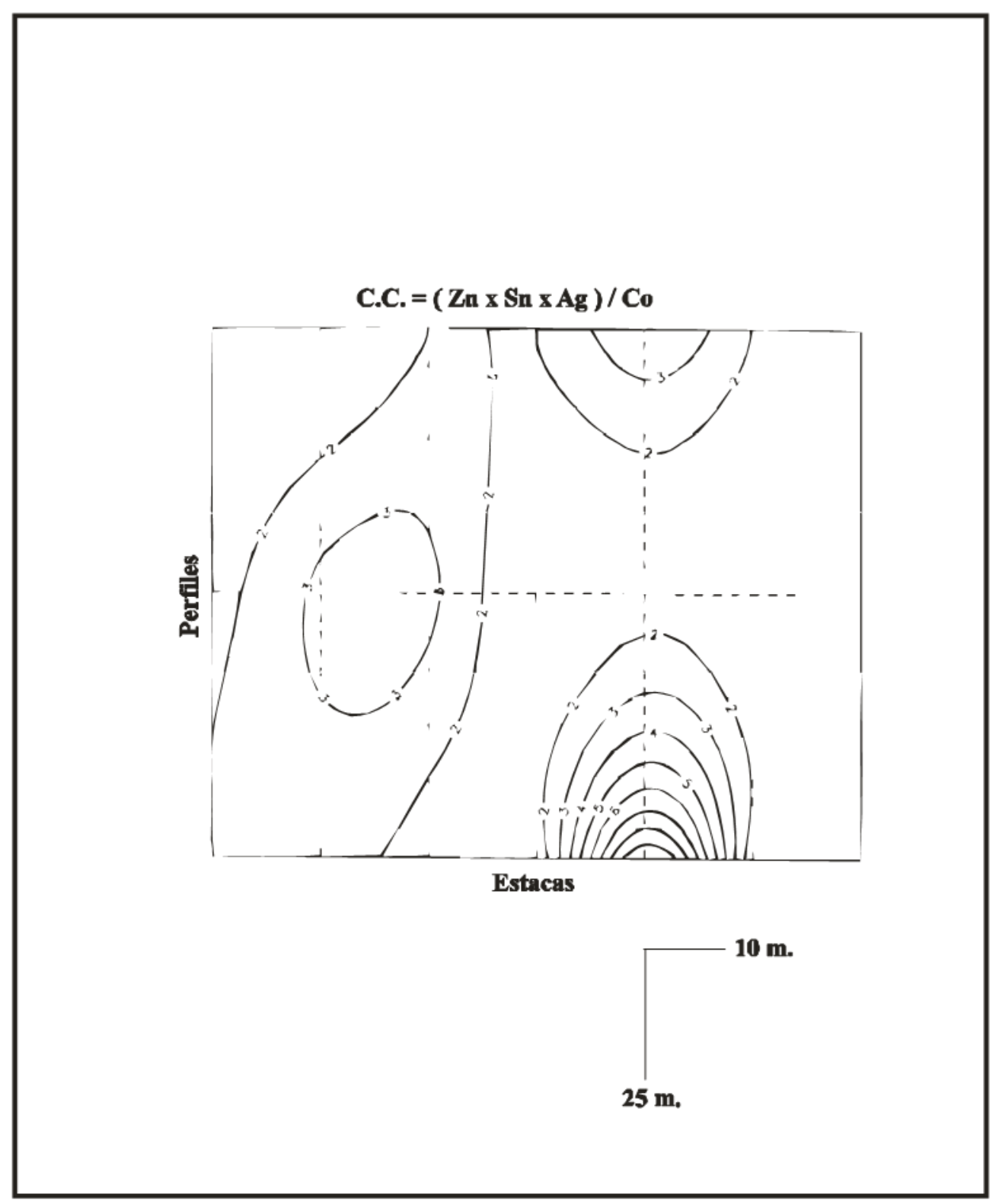

Figura 10. El C.C. (Zn $x$ Sn $\times A g) / C o$, permite el mapeo de la zona mineralizada, incluyendo no sólo el área de las "menas negras", sino también el área de desarrollo de la zona de alteración. 


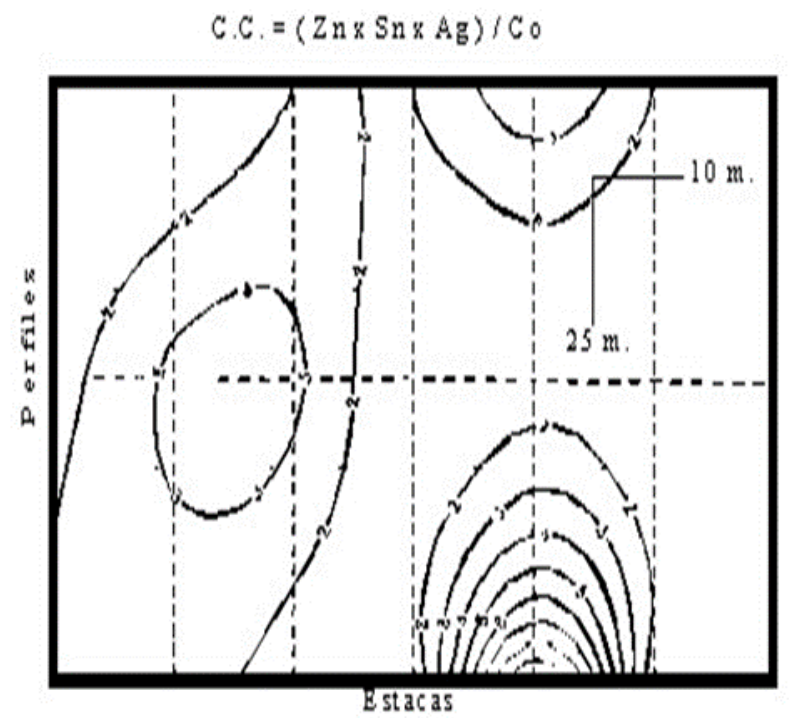

Figura 11. Representación en el plano del C.C. (Zn x Sn x Ag) / Co .

El siguiente paso consistió en comparar los resultados geoquímicos de la zona mineralizada con la zona de alteración (Tablas 5 y 6), con vistas a tratar de incrementar la selectividad del C.C. establecido.

Tabla 5. Características geoquímicas de la zona mineralizada Total de observaciones: 6.

\begin{tabular}{lrrrrrrr} 
& \multicolumn{1}{c}{$\mathbf{0 / 8}$} & \multicolumn{1}{c}{$\mathbf{0 / 1 0}$} & \multicolumn{1}{c}{$\mathbf{2 / 8}$} & \multicolumn{2}{c}{$/ \mathbf{1 0}$} & \multicolumn{4}{c}{$\mathbf{8}$} & $\mathbf{4 / 1 0}$ & Media \\
\hline $\mathrm{P}$ & 200.0 & 80.0 & 60.0 & 80.0 & 80.0 & 80.0 & 96.7 \\
$\mathrm{~Pb}$ & 3.0 & 2.0 & 2.0 & 3.0 & 2.0 & 1.0 & 2.2 \\
$\mathrm{Sn}$ & 2.0 & 2.0 & 1.0 & 1.0 & 2.0 & 1.0 & 1.5 \\
$\mathrm{Ag}$ & 0.1 & 0.1 & 0.1 & 0.1 & 0.2 & 0.1 & 0.1 \\
$\mathrm{Ni}$ & 30.0 & 40.0 & 10.0 & 30.0 & 6.0 & 6.0 & 20.3 \\
$\mathrm{Cu}$ & 100.0 & 100.0 & 80.0 & 100.0 & 80.0 & 60.0 & 86.7 \\
$\mathrm{Co}$ & 10.0 & 20.0 & 10.0 & 30.0 & 8.0 & 6.0 & 14.0 \\
$\mathrm{Zn}$ & 200.0 & 200.0 & 200.0 & 200.0 & 200.0 & 100.0 & 183.3 \\
$\mathrm{Mn}$ & 1000.0 & 1000.0 & 600.0 & 800.0 & 300.0 & 100.0 & 633.3 \\
$\mathrm{~V}$ & 100.0 & 100.0 & 80.0 & 100.0 & 80.0 & 80.0 & 90.0 \\
$\mathrm{Ga}$ & 20.0 & 20.0 & 20.0 & 20.0 & 20.0 & 8.0 & 18.0 \\
\hline
\end{tabular}


Tabla 6. Características geoquímicas de la zona de alteración. Total de observaciones: 6.

\begin{tabular}{lrrrrrrr} 
& \multicolumn{1}{c}{$0 / 4$} & \multicolumn{1}{c}{$0 / 6$} & \multicolumn{1}{c}{$2 / 4$} & \multicolumn{1}{c}{$2 / 6$} & \multicolumn{1}{c}{$4 / 4$} & \multicolumn{1}{c}{$4 / 6$} & \multicolumn{1}{c}{ Media } \\
\hline $\mathrm{P}$ & 100.0 & 100.0 & 80.0 & 100.0 & 100.0 & 80.0 & 93.3 \\
$\mathrm{~Pb}$ & 4.0 & 1.0 & 2.0 & 1.0 & 1.0 & 1.0 & 1.7 \\
$\mathrm{Sn}$ & 2.0 & 2.0 & 2.0 & 1.0 & 1.0 & 1.0 & 1.5 \\
$\mathrm{Ag}$ & 0.1 & 0.1 & 0.1 & 0.1 & 0.1 & 0.1 & 0.1 \\
$\mathrm{Ni}$ & 20.0 & 30.0 & 20.0 & 8.0 & 6.0 & 6.0 & 15.0 \\
$\mathrm{Cu}$ & 80.0 & 100.0 & 80.0 & 80.0 & 80.0 & 60.0 & 80.0 \\
$\mathrm{Co}$ & 10.0 & 10.0 & 10.0 & 8.0 & 6.0 & 6.0 & 8.3 \\
$\mathrm{Zn}$ & 100.0 & 100.0 & 200.0 & 100.0 & 100.0 & 100.0 & 116.7 \\
$\mathrm{Mn}$ & 800.0 & 1000.0 & 600.0 & 400.0 & 200.0 & 200.0 & 533.3 \\
$\mathrm{~V}$ & 100.0 & 100.0 & 80.0 & 80.0 & 80.0 & 80.0 & 86.7 \\
$\mathrm{Ga}$ & 20.0 & 20.0 & 20.0 & 20.0 & 10.0 & 10.0 & 16.7 \\
\hline
\end{tabular}

Para ello se empleó el criterio de analogía de Student (7), asumiendo como valor crítico $t_{\text {cr }}=2$ (Kazhdan, 1979).

Ecuación (7) $t=\frac{|M x-M y|}{\sqrt{\left(\frac{S x^{2}}{n_{x}}+\frac{S y^{2}}{n_{y}}\right)}}$

donde,

$t \quad$ - criterio de analogía de Student

Mx - valor medio de la muestra X

Sx - desviación standard de la muestra X

$n x \quad$ - volumen de la muestra $\mathrm{X}$

My - valor medio de la muestra Y

Sy - desviación standard de la muestra Y

ny - volumen de la muestra Y

Los resultados de dicha comparación (tabla 7) explican claramente el por qué ambas zonas aparecen mapeadas con el mismo coeficiente correlacional, pues -a excepción del cinc- todos los demás elementos presentan contenidos análogos en ambas zonas. Análisis correlacionales para cada grupo mostraron relaciones así mismo similares, por lo cual es imposible -e innecesariodefinir C.C. independientes para ambas zonas. 
Tabla 7. Comparación entre las características geoquímicas de la zona mineralizada y de la zona de alteración por medio del criterio de Student.

\begin{tabular}{ccc} 
Elemento & t-Student & Análogos? \\
\hline $\mathbf{A g}$ & 1.22 & $\mathrm{Si}$ \\
$\mathbf{C o}$ & 1.48 & $\mathrm{Si}$ \\
$\mathbf{C u}$ & 0.79 & $\mathrm{Si}$ \\
$\mathbf{G a}$ & 0.37 & $\mathrm{Si}$ \\
$\mathbf{M n}$ & 0.49 & $\mathrm{Si}$ \\
$\mathbf{~ N i}$ & 0.74 & $\mathrm{Si}$ \\
$\mathbf{P}$ & 0.16 & $\mathrm{Si}$ \\
$\mathbf{P b}$ & 0.88 & $\mathrm{Si}$ \\
$\mathbf{S n}$ & 0.00 & $\mathrm{Si}$ \\
$\mathbf{V}$ & 0.54 & $\mathrm{Si}$ \\
$\mathbf{Z n}$ & 2.89 & $\mathrm{No}$ \\
\hline
\end{tabular}

El último paso consiste en determinar a partir de qué valor los C.C. son perspectivos, lo cual se calcula por la combinación de los valores máximos y mínimos posibles para cada C.C. según la ecuación (8).

Ecuación (8) $X_{\text {c.c. }}=\frac{1}{n} *\left(X_{\text {c.c. } \max }+X_{\text {c.c. } \min }\right)$

donde,

$X_{c . c .} \quad$ - límite inferior de los valores perspectivos.

$X_{\text {c.c.max }}$ - valor máximo posible para el C.C. analizado ${ }^{1}$

$X_{\text {c.c.min }}$ - valor mínimo posible para el C.C. analizado

$n \quad$ - factor de perspectividad.

En nuestro caso, teniendo en cuenta la relativa gran diferencia entre los valores máximos y mínimos de los C.C. analizados, "n" se tomó igual a 4. De esta forma se determinaron los siguientes límites de valores perspectivos.

a) $\frac{N i * C o * M n * G a * V * C u}{P * 10^{8}}=5$

b) $\frac{Z n * S n * A g}{C o}=5$

${ }^{1}$ Dicho valor se obtiene asumiendo para los elementos del numerador sus valores máximos, combinado con los valores mínimos en el denominador. Para el valor mínimo, se procede de forma inversa.

19 


\section{Conclusiones y recomendaciones}

Tanto las características geoquímicas y mineralógicas, como las tectónicas y geológicas del Sector "Los Tramojos", coinciden con las de los yacimientos vulcanógeno-exhalativos submarinos del Tipo Kuroko, de no contar con la ausencia de un vulcanismo ácido que, si bien no es condición obligatoria para este tipo de yacimientos, sí es su ambiente más corriente.

Dada la importancia económica de este tipo de yacimientos, se recomienda un estudio completo del área, apoyado por métodos geofísicos y geoquímicos que permitan no sólo la delimitación del cuerpo detectado, sino también la ubicación de otros similares, posiblemente asociados igualmente a las elevaciones del terreno.

Particularmente en la interpretación de los muestreos metalométricos se recomienda emplear el coeficiente correlacional $(\mathrm{Zn} * \mathrm{Sn} * \mathrm{Ag}) / \mathrm{Co}>5$, para el mapeo de las zonas potencialmente meníferas.

\section{Agradecimientos}

El autor agradece el apoyo recibido por el Departamento de Ciencias de La Tierra y El Espacio de la Universidad McGill de Montreal y muy especialmente por parte de su Centro de Cálculo, para la edición tanto del texto como de las figuras que lo acompañan.

Así mismo, quisiera reconocer la ayuda brindada por los técnicos Ramón Valdivia y Ramón Arencibia en la ejecución de los trabajos de campo, y al Laboratorio Provincial de la Expedición Geológica de Santa Clara, por la pronta entrega de los resultados.

\section{References}

Academia de Ciencias de Cuba y academia de Ciencias de Polonia, 1970, Levantamiento Geológico 1:500 000 de Cuba, La Habana: Archivo Geológico.

Cox, D. P. and Singer, D. A., 1986, ed. Mineral Deposits Models. Washington: United States Printing Office, $379 \mathrm{p}$.

Evans, A.M., 1987, An Introduction to Ore Geology. 2nd. edition. Oxford:Blackwell Scientific Publications, $358 \mathrm{p}$.

Kazhdan, A.B. et al., 1979, Matematicheskoie modelirovanie v geologii i razvedki poleznij iskopaemij [Modelaje matemático en la Geología y la Búsqueda de Yacimientos Minerales]. Moscú: Nedra, 168 p.

Millan García, E., 1989, Análisis formacional de la Cuenca Central de Cuba. En Revista Tecnológica de Cuba, Vol. XIX, No. 1, pp.16-25.

Ministerio de la Industria Básica, 1988, Informe del Levantamiento Geológico 1:50 000 Escambray-II. La Habana: Archivo Geológico. 
Rotes, H.J. and LANGE, H., 1972, Geochemical Tables. Amsterdam-London-New York: Elsevier Publishing Company, pp. 234 - 236.

Valls Álvarez, R. A. y NUÑEZ, F., 1989, Los métodos geoquímicos en la búsqueda de talco en el macizo metamórfico del Escambray. En Revista Tecnológica Cubana, Vol. XIX, No. 1, pp. 9 15.

Valls Álvarez, R. A., 1993, Tercera versión de las fichas codificadas; ventajas de su uso. Revista E.A.F.I.T., Medellín, Colombia.

Wedepohl, K.H. ed., 1978, Handbook of Geochemistry. Germany: Springer-Verlag, Berlín, Tomo II-2 al Tomo II-5. 